\title{
Investigation to Increase the Welding Joint Area with Modulated Laser Beam Welding over Gap
}

\author{
Woo-Sik Chung, Andre Haeusler, Alexander Olowinsky, Arnold Gillner, Reinhart Poprawe \\ Fraunhofer Institute for Laser Technology ILT, Steinbachstr. 15, 52074 Aachen, Germany \\ E-mail: woo-sik.chung@ilt.fraunhofer.de
}

\begin{abstract}
Due to the increasing power level of semiconductor components such as LEDs and IGBTs, larger connection areas are required in the near future, which should be also stable at high temperatures. However, these requirements cannot be realized by conventional joining processes such as ribbon bonding and soldering for contacting semiconductor components. Traditional welding methods, such as laser beam welding, are not used because the conventional process approaches induce thermal stress beyond the destruction threshold of sensitive substrate materials. The Laser Impulse Metal Bonding (LIMBO) realizes a laser-gap welding process between a $200 \mu \mathrm{m}$ thick copper sheet and a $105 \mu \mathrm{m}$ thick copper plating board without damaging the underlying PCB. The central approach of the LIMBO process is the modulation of the focus diameter to adjust the laser beam intensity on the workpiece. This results in melting, deflection and contacting the upper joining partner with the lower one. However, this method is currently only limited to spot welding. The aim of this paper is to expand the LIMBO process to increase reproducibility by using pre-pulsing method and the process stability with controlling the energy input and the beam modulation. Based on this expansion, the spot welding process is transferred to a linear connection between the two joining partners with overlap pulsed welding method over a weld joint length over a $l=1 \mathrm{~mm}$ without destructing the underlying thermal sensitive substrate.

DOI: 10.2961/jlmn.2018.02.0011
\end{abstract}

Keywords: laser gap welding, beam modulation, LIMBO, reproducibility, overlap pulsed welding

\section{Introduction}

The demands on high power systems such as those used in electric vehicles or power electronic devices increased throughout the years since the energy transition. In order to meet technological and economical requirements for manufacturing high power systems in near future, a promising joining technology which allows high thermal stability up to $\mathrm{T}=220^{\circ} \mathrm{C}$ and large weld joint area for low electrical resistance is essential [1]. To realize electrical interconnection, conventionally used joining methods in mass production are brazing, wire (ribbon) bonding and silver sintering. However, those conventional technologies are limited due to low long-term stability for the brazing [2], small joint area as well as limited combination of joining material for wire bonding [3] and the risk of damaging underlying substrate because of high pressure during silver sintering process [4].

Britten et al. proposed the Laser Impulse Metal Bonding (LIMBO) process which poses to be a new alternative joining method to fulfill the demands of joining technology in terms of high thermal stability without destruction of the underlying thermally sensitive substrate [5]. The LIMBO process is based on spike welding method, which was first introduced by Dijken et al. to bridge gaps between steel construction assemblies [6].

Yet, the Limbo process has the limitation of the weld joint area due to the nature of spot welding method. Hence, to expand the possible application for LIMBO process, it is necessary to enhance the welding joint area for the power electronic components, which is used under high current application by lowering the electrical resistance through enlarging the weld joint area. By achieving expanded weld joint area it is also expected that the mechanical strength in terms of shock test, vibration test, and pull \& peel test would be improved as well.

In this paper, the reproducibility and the reliability of copper welding using the LIMBO process is discussed. Based on this discussion, the expansion of weld joint area is carried out to achieve high thermal stability and wide weld joint area between massive copper interconnector and thin copper layer on a thermally sensitive substrate.

\section{State of the art}

\subsection{Laser impulse metal bonding}

In order to minimize the thermal stress to thermally sensitive substrates, only limited energy of laser beam should be allowed into the underlying joining partner. The LIMBO process allows to control the residual time of the melt on the underlying copper layer with modulated laser beam during the welding process. The LIMBO process is divided into three stages (figure 1) and carried out with $\mathrm{Nd}$ :YAG laser beam source with maximum power output of $\mathrm{P}=3 \mathrm{~kW}$. [7]

In the first stage, which is called pre-heating stage, the interconnector is locally exposed to laser beam until it is melted through by heat conduction (figure 1 left). After this stage the increased power modulation of laser beam, magnifies the metal vapor plume at upper surface of the molten copper substrate. Due to the recoil pressure of metal vapor 
plume on upper surface of the locally molten copper, the melt pool is accelerated to the underlying copper layer (figure 1 middle). This stage is called deflection stage. As soon as the accelerated melt contacts the underlying copper layer, it starts locally to heat up due to heat conduction. At the joining stage of the LIMBO process the melt pool of the upper joining partner penetrates into the copper layer and the two joining partners are welded together (figure 1 right). This novel method offers a possibility to join a thin copper layer on a thermally sensitive substrate with a thick interconnector by a weld joint

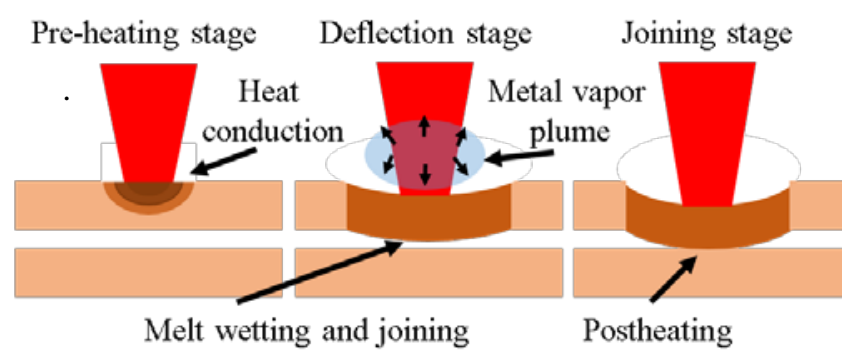

Fig. 1 LIMBO process [7]

The final state after a successful LIMBO process is a weld joint between both joining partners over a gap without destructing the underlying substrate and a solidified melt of the interconnector. The diameter of solidified melt shows greater value compared to that of the weld joint due to the longer exposure to laser beam and heat conduction in preheating stage. This final state of the LIMBO process is also the initial state for weld joint area expansion. The top view after a LIMBO process is shown in figure 2.

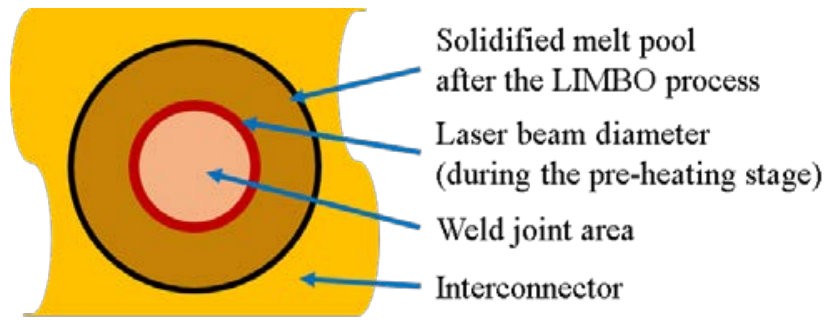

Fig. 2 Schematic representation after the LIMBO process

\subsection{Overlap pulsed welding}

As for the approach to expand the weld joint area of the LIMBO process, overlap pulsed welding is purposed, since it is a spot welding method. In overlap pulsed welding a portion of the previous spot is reheated and remelted. In to order analyze the behavior of overlap pulsed welding, Torkamany et al. suggested the overlapping factor $\mathrm{O}_{\mathrm{f}}$ with the formula

$$
\mathrm{O}_{\mathrm{f}}=[1-(\mathrm{v} / \mathrm{f}) /(\mathrm{D}+\mathrm{v} \times \mathrm{t})] \times 100
$$

dependent on the laser spot diameter $\mathrm{D}$, welding speed $\mathrm{v}$, frequency $\mathrm{f}$ and pulse duration $\mathrm{t}$ for welding st14 sheets with thickness $\mathrm{h}=0.7 \mathrm{~mm}$ by using $\mathrm{Nd}$ :YAG laser beam source. According to the examination, the overlap pulsed welding is conducted with the pulse duration $\mathrm{t}=6 \mathrm{~ms}$ and the frequency $f=20 \mathrm{~Hz}$. As for the laser spot size on the work piece $\mathrm{D}=1.2 \mathrm{~mm}$ is applied with varying welding velocities between $\mathrm{v}=[5 ; 11.5] \mathrm{mm} / \mathrm{s}$. [8]

To expand the weld joint of the LIMBO process, a melt pool should be deflected adjacent or partly adjacent to an already existing weld joint. Due to greater pulse duration of the LIMBO process with $\mathrm{t}=20 \mathrm{~ms}$ in combination with much smaller laser spot size in region $\mathrm{D}=300 \mu \mathrm{m}$, only limited laser beam frequency and welding speed can be applied for overlap pulsed welding with the LIMBO process. Also, the divided stages of the LIMBO process should also taken into account for the overlap pulsed welding.

In addition, the heat accumulation during the overlap welding should be considered. The initial state for overlap welding is joining partners that are already connected and this state conflicts with the idea of the LIMBO process, whereas the upper and lower joining partners are separated to minimize the thermal stress on thermally sensitive substrate during the pre-heating stage. So, throughout the overlap welding the underlying joining partner is under thermal conduction and convection stress during the total overlap welding process.

\section{Experimental set up and methods}

\subsection{Experimental set up}

In order to investigate the reproducibility of each LIMBO process every experiment was carried out five times repeatedly. As for the upper joining partner a $\mathrm{CuFe}_{2} \mathrm{P}$ material with thickness $\mathrm{h}=200 \mu \mathrm{m}$ with area dimension $10 \mathrm{~mm} \times 50 \mathrm{~mm}$ is selected. The underlying joining partner is a glass fiber reinforced epoxy resin with copper layer with a thickness $\mathrm{h}=100 \mu \mathrm{m}$. The copper layer surface is coated with a nickel-gold alloy in order to prevent the copper metal from the oxidization in surrounding atmosphere. This nickel-gold alloy has a thickness $\mathrm{h}=6 \mu \mathrm{m}$. The gap between both joining partners is defined as $\mathrm{a}=100 \mu \mathrm{m}$. The schematic representation of the experimental set up is shown in figure 3 .

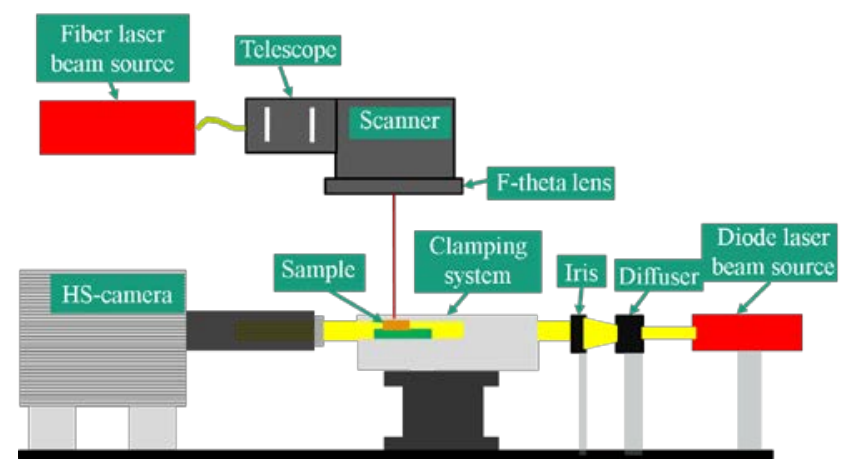

Fig. 3 Schematic representation of the experimental set up

For this study, the welding process is carried out by a single-mode fiber laser with maximum power $\mathrm{P}=1 \mathrm{~kW}$ which has a wavelength of $\lambda=1070 \mathrm{~nm}$ and a spot diameter $\mathrm{D}=36 \mu \mathrm{m}$ at the focal position on a workpiece. The $\mathrm{f}$ theta lens with a focal length $\mathrm{f}=163 \mathrm{~mm}$ is used.

A high speed camera (HS-camera) was used to capture melt pool dynamics between both joining partners in order to examine the deflected melt during the deflection stage. 
Also an assistance of strong light source is required to gain a sharp shadow projection of melt deflection in high resolution. For this purpose a diode laser beam with wavelength $\lambda=810 \mathrm{~nm}$ with maximum power $\mathrm{P}=200 \mathrm{~W}$ is used.

\subsection{Temporal spot diameter modulation}

To modulate the laser beam in the deflection stage of the LIMBO process and for expanding the weld joint area with overlap pulsed welding, a temporal spot diameter modulation was conducted to deflect the melt to underlying substrate [9]. In order to modulate the spot diameter temporally, a set of lenses was placed between laser beam collimator and scanner. Through a temporally adjusting focal lens, the focal position is varied in z-direction on the workpiece whereas the distance from f-theta lens to the specimen stays constant (figure 4).

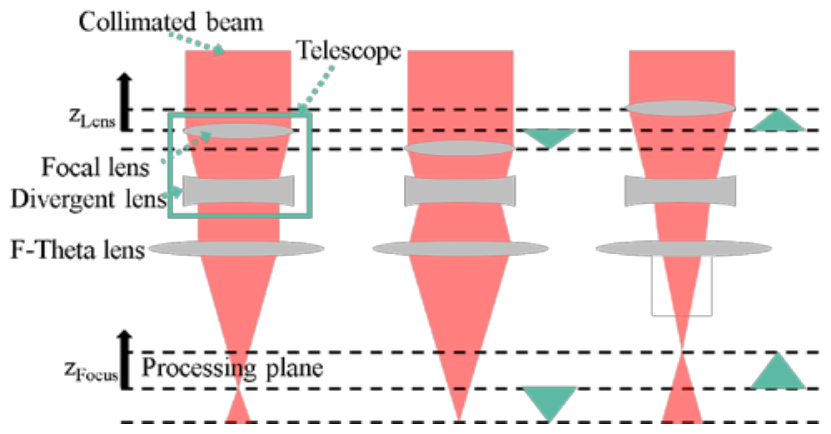

Fig. 4 Principle of temporal focal position adjustment [9]

The adjustment of the focal lens can be done from the zero position up to $\mathrm{z}=2 \mathrm{~mm}$ in positive and negative directions with $\Delta \mathrm{z}=0.1 \mathrm{~mm}$ steps. Since the LIMBO process is executed in one single pulse, the intensity of laser beam increases over time in correlation with the adjusting movement of the focal lens. Due to the given setting of lenses, the emitting time of laser beam is extended by $\mathrm{t}=1 \mathrm{~ms}$ for every $\Delta \mathrm{z}=0.1 \mathrm{~mm}$ adjustment of focal lens. Therefore the extended duration of laser beam irradiation during the focal lens adjustment leads to further energy input to the specimen. The laser beam energy accessed in the work specimen increases with longer duration of deflection stage, whereas the temporal increasement of laser beam energy is not given, since the laser beam power stays constant over the whole pulse duration. However, the intensity of laser beam increases temporally with the growing range of adjustment during the deflection stage.

\subsection{Method to investigate the reproducibility}

The reproducibility of the pre-heating stage is investigated by comparison of the geometrical value. To control the lens-like melt formation reliability and the diameter of weld spot through the heat conduction welding, three different surface conditions were investigated. The investigated surface conditions are: pre-oxidized surface through a short laser beam pulse, mechanically roughened surface by sand-blasting and rolled surface as a reference.

The melt pool created only by the heat conduction welding leads to a heat accumulation effect at the bottom side of substrate if the substrate has a limited thickness.
Due to this limitation, the modification of the melt pool geometry occurs at the bottom side of the substrate. Therefore, in terms of heat conduction welding the melt pool diameter at the lower side of substrate $d_{b}$ cannot be bigger than the melt pool diameter at upper side of substrate $d_{t}$. The ratio between the those melt pool diameters, the melt pool diameter ratio $\zeta=d_{b} / d_{t}$ is introduced to investigate the reproducibility of heat conduction welding.

In the deflection stage, the melt deflection is observed under separated phases: 'Start of deflection', 'Maximum deflection' and 'End of deflection'. The duration of each phase in the deflection stage is compared dependent to focal lens adjustment value. The time slope between each phase in deflection stage indicates the duration of each phase. When the time slope between each phase varies, the transition to next phase is influenced and consequently the energy input to the melt pool changes. Hence, the main goal during the deflection stage is to minimize the energy input and obtain stable values with the lowest standard deviation value at each phase.

\subsection{Method to expand the weld joint area}

The investigation for magnifying mechanism of the weld joint diameter through the overlap pulsed welding requires a new definition of the overlapping factor due to circumstances described in sub-section 2.2. Conventionally, the overlapping factor from [8] is calculated by the overlapped area between each non-varying laser beam spot in welding direction. By conducting the LIMBO process with temporal modulation of spot diameter, the varying laser beam spot diameter cannot be included in this formula.

As an approach on the overlap pulsed welding with the LIMBO process, the geometrical value is defined as reference factor for determining the overlapping factor. Outgoing from the solidified melt pool diameter on upper joining partner $d_{t}$, the pulse distance $x_{\text {Pulse }}$ for overlapping factor can be calculated. The temporal terms are not included in the definition of overlapping factor, due to possibility of verifying duration of overlap pulsed welding dependent on the number of exercised overlap pulsed welding. Also with the laser beam constantly moving in the welding direction, the LIMBO process cannot be applied correctly, since the lens-like melt formation is not guaranteed because of insufficient duration of the pre-heating stage. In addition, an aggressive steering of the focal lens in the deflection stage may lead to oscillation even after reaching the adjustment distance. In order to guarantee the achievement of adjusted focal lens distance a sufficient cool time between each overlap welding is integrated. Thus, a novel definition of the overlapping factor is shown in equation

$$
\mathrm{O}_{\mathrm{f}}=\left(1-\mathrm{XPulse}_{\text {Pe }} / \mathrm{d}_{\mathrm{t}}\right) \times 100
$$

which is dependent on the distance between each conducted pulse Xpulse solidified melt pool diameter on upper joining partner $d_{t}$ of the single LIMBO process in this phase of examination. In order to investigate characteristics of the overlap pulsed welding with the LIMBO process by using this definition, a reproducible geometrical value of the LIMBO process is essential. The deviation of geometrical value can lead to manipulation of calculated overlapping factor. After reaching reproducible LIMBO process, 
three representative cases of the overlap pulsed welding is demonstrated to investigate the behavior of the LIMBO process during the overlap pulsed welding.

\section{Reproducibility of the copper welding}

\subsection{Geometrical value}

The initial stage of the joining process throughout the laser-gap welding is after forming a lens-like melt at the lower side of the interconnector which is deflected to the underlying substrate. The stable formation of the lens-like melt is essential in performing laser-gap welding. To achieve this lens-like melt, the interconnector is welded dominantly by heat conduction. When a keyhole occurs the laser beam can be transmitted though the keyhole and directly radiated onto the underlying substrate, uncontrolled, and therefore the substrate can be thermally damaged. Furthermore, the thermally sensitive substrate can be thermally damaged due to the uncontrolled residence time of the melt. In addition, the keyhole welding can cause melt droplets and the melt pool volume reduces for the actual deflection phase and results in smaller weld joint diameter.

The heat conduction welding of the pre-heating stage is investigated by comparing the solidified melt pool diameter ratio between pre-oxidized, roughened and rolled surfaces to obtain reliable lens-like melt at lower side of the interconnector. The pre-oxidization is performed with a short laser beam pulse with following process parameter: power $\mathrm{P}=500 \mathrm{~W}$, spot size $\mathrm{D}=60.4 \mu \mathrm{m}$, duration $\mathrm{t}=400 \mu \mathrm{s}$ and the roughened surface is sand-blasted with following process parameter: pressure $\mathrm{p}=1.5 \mathrm{bar}$, angle of gun to the specimen $\gamma=45^{\circ}, \mathrm{t}=60 \mathrm{~s}$ and grain size of sand $\mathrm{d}=40 \sim 70 \mu \mathrm{m}$. The absorption rate of each surface condition at wavelength $\lambda=1070 \mathrm{~nm}$ is summarized in table 1 .

Table 1 Absorption rate $\alpha$ of different surface conditions at wavelength $\lambda=1070 \mathrm{~nm}$

\begin{tabular}{cc}
\hline Pre-oxidized & $5.52 \%$ \\
Roughened & $19.82 \%$ \\
Rolled & $1.02 \%$ \\
\hline
\end{tabular}

This experiment was carried out also with identical process parameters for each surface condition five times to determine the geometrical deviation value (figure 5). The process parameters were determined with preliminary experiments.

The standard deviation value for the melt pool diameter at lower side of upper substrate $d_{b}$ is for all three surface conditions relatively small compared to that melt pool diameter at upper side of upper substrate. The phenomenon can be explained by the rapid enhancement of absorption rate of the copper material between the solid and liquid state. Also, the thermal conductivity of copper material rapidly drops during the phase transition from solid state to liquid state. [10]

The diverse time period for absorbing laser beam on the copper substrate leads to different moment of reaching melting temperature. Due to five times higher absorption rate of copper in liquid state than in solid state, the duration of pre-heating stage before reaching the melting temperature of copper material gives a substantial effect on the absolute amount of absorbed laser beam energy. The welding process which reaches the melting temperature of copper substrate faster, absorbs larger laser beam energy amount. In addition, the reduced thermal conductivity favors the heat accumulation in the melt. Thus, the lower side of solidified melt pool diameter can have relatively higher deviation. On the other hand, the upper side of solidified melt pool diameter is affected less by the absorption rate of copper substrate because the surface of the melt pool is directly irradiated by the laser beam during the entire pre-heating stage. Therefore, the upper side of solidified melt pool of all three surface conditions have sufficient energy supply to reach their maximum value $d_{t}$.

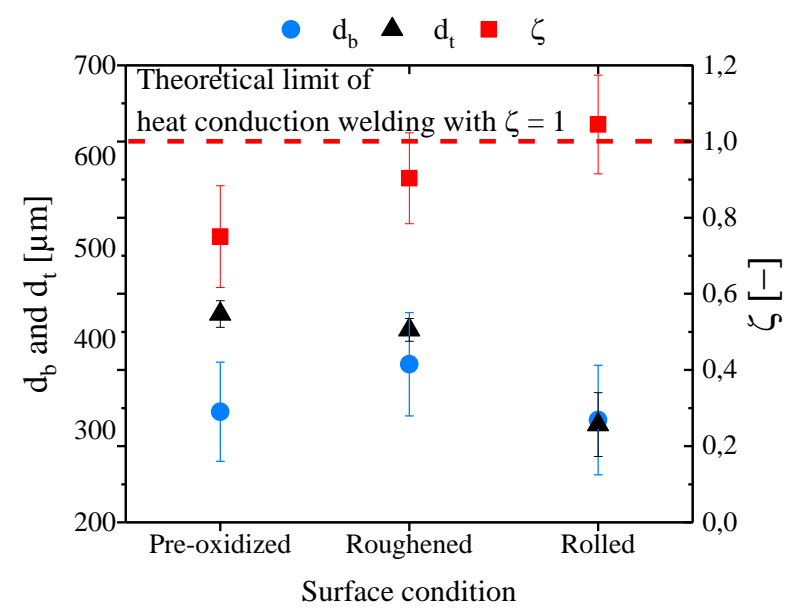

Fig. 5 Geometrical value after the pre-heating stage

As for the rolled surface, the melt pool diameter ratio is above the theoretical limit for heat conduction welding and purpose inappropriate results in forming a stable lens-like melt after the pre-heating stage. In comparison to the rolled surface, the average value of solidified melt pool diameter ratio of roughened and pre-oxidized surfaces are suitable for heat conduction welding in order to form a lens-like melt. The average value of the melt pool diameter ratio is for roughened copper surface $\zeta_{\text {rough }}=0.903$ the highest and shows the smallest standard deviation with $\sigma_{\text {rough }}=0.12$. Despite this advantageous geometrical value for roughened copper surface, there is a risk of exceeding the boundary of heat conduction welding when taking into account the deviation value in sand-blasting process.

Due to the suitable geometrical value of the preoxidized surface of the copper substrate regarding the LIMBO process, an additional stage should be included before the pre-heating stage. The short laser beam pulse to locally pre-oxidize the interconnector is defined as the prepulse stage.

\subsection{Melt deflection}

The reproducibility of the LIMBO process in deflection stage is discussed in this section. The primary aim of deflection stage is to deflect the melt over a defined gap $\mathrm{a}=100 \mu \mathrm{m}$ between both joining partners. Through highspeed camera videography the suitable adjustment range for focal lens is determined as $\Delta \mathrm{z}=[0.4 \mathrm{~mm}$, 
$0.5 \mathrm{~mm}, 0.6 \mathrm{~mm}$ ] in this regard. The melt deflection of the LIMBO process captured with the high-speed videography is shown in figure 6 with the description of the shadow projection for this paper.

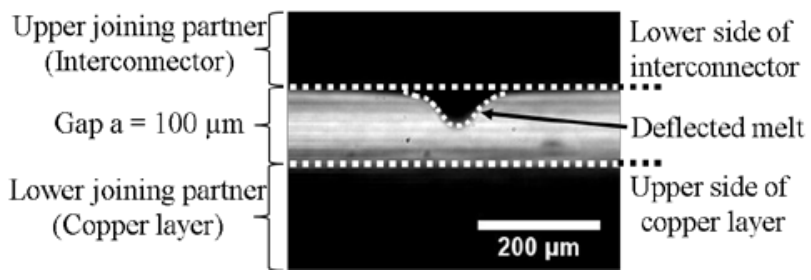

Fig. 6 High-speed camera videography during deflection stage

In order to investigate the deflection stage, the deflection stage is separated in three different phases: "Start of deflection", "Maximum deflection" and "End of deflection" (refer to sub-section 3.2). The phase "Start of deflection" is defined when the melt starts to deflect after a certain time during the deflection stage due to increasement of the laser beam intensity (figure 7 left). After continuously increasing value of the laser beam intensity due to the adjustment of the focal lens, the melt reaches its maximum deflection value and this moment is defined as the "Maximum deflection" (figure 7 middle). When the laser beam radiation emission is determined, the melt dynamic starts to relax and the "End of deflection" is defined when the melt is completely relaxed (figure 7 right). The blind welding process is proposed to observe each phase of the deflection stage. Otherwise the deflected melt during the deflection stage drops on the surface of the underlying copper layer and leads to a wetting process of the melt and copper layer. The each phase of the deflection stage (blind welding) is shown in figure 7 and the description of the shadow projection is equal to the description in figure 6 .
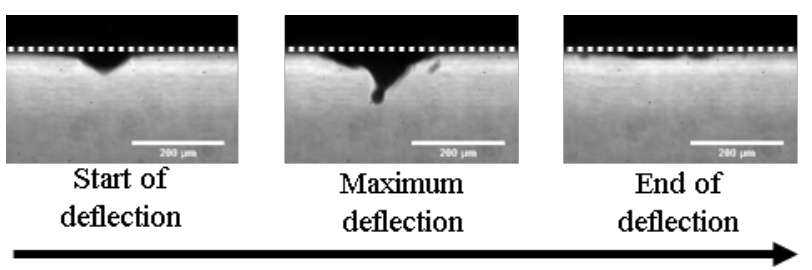

Time flow in the deflection stage

Fig. 7 Time development of the melt deflection

The reproducibility of the deflection stage is investigated in blind welding process to obtain deflected melt behavior in correlation with different focal lens adjustment value. The influence of temporal modulated laser beam on deflection melt during deflection phase is shown in figure 8 .

The standard deviation value from each phase in the deflection stage has been distinguished. In initial phase, the focal lens adjustment $\Delta \mathrm{z}=0.4 \mathrm{~mm}$ represents the highest deviation value at 'Start of deflection' with $\sigma_{t}= \pm 1.13 \mathrm{~ms}$. The focal lens adjustment $\Delta \mathrm{z}=0.5 \mathrm{~mm}$ and $0.6 \mathrm{~mm}$ show that the phase 'Start of deflection' phase occurs nearly at the same average time value. However, the standard deviation value of the focal lens adjustment $\Delta \mathrm{z}=0.5 \mathrm{~mm}$ shows the most stable value compared to other adjustment value with $\sigma_{t}= \pm 0.48 \mathrm{~ms}$ respectively. As for the 'Maximum deflection' phase the adjustment value $\Delta \mathrm{z}=0.5 \mathrm{~mm}$ shows the lowest standard deviation value with $\sigma_{t}= \pm 0.27 \mathrm{~ms}$ and represents the highest stability concerning melt deflection over a defined gap distance. In 'End of deflection' phase the standard deviation values are comparable to each other.

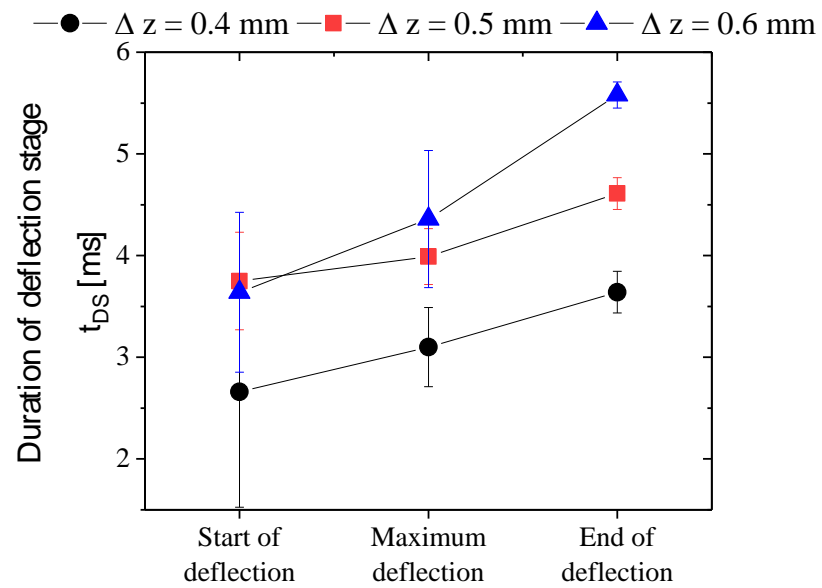

Fig. 8 Melt deflection correlation to focal lens adjustment

Under the assumption that the joining procedure begins at the maximum value of deflection, the time duration between the phases 'End of deflection' and 'Maximum deflection' is decisive in determining thermal stress on the lower joining partner through the heat conduction. By comparing the time slope between both phases for three adjustment values, an increasing tendency of slope with higher adjustment range is observed. However, the difference between $\Delta \mathrm{z}=0.4 \mathrm{~mm}$ with $\Delta \mathrm{t}=0.53 \mathrm{~ms}$ and $\Delta \mathrm{z}=0.5 \mathrm{~mm}$ with $\Delta \mathrm{t}=0.62 \mathrm{~ms}$ is $\Delta \mathrm{t}=90 \mu \mathrm{s}$, which is within range of the standard deviation value for both adjustment of the focal position.

To summarize the deflection stage for the LIMBO process, the adjustment value $\Delta \mathrm{z}=0.5 \mathrm{~mm}$ is chosen due to shortest duration between phases 'End of deflection' and 'Maximum deflection'. Therefore, the expected minimal input of thermal stress to lower joining partner during the deflection stage is the lowest for $\Delta \mathrm{z}=0.5 \mathrm{~mm}$. Furthermore, the lowest standard deviation value in phase 'Maximum deflection' leads to the most suitable and reproducible results.

\section{Expansion of the weld joint area}

Identical to the single LIMBO process, the process to expand the weld joint area with overlap pulsed welding is also divided in three stages: pre-heating stage, deflection stage and joining stage. Throughout the preliminary experiments on overlap pulsed welding, the pre-pulsing stage did not influence the overlap pulsed welding procedure due to the surface oxidization and solidified melt pool of the interconnector. To investigate the behavior of the LIMBO process during the overlap pulsed welding, the pre-heating and deflection stages are considered separately in terms of the overlapping factor and the number of applied overlap pulsed welding. In addition, the destructing threshold for thermal sensitive substrate should be also taken into account in the overlap pulsed welding process. 
With established process parameters, four overlap pulsed welding processes were continuously conducted to expand the weld joint area which is dependent to various overlapping factors.

\subsection{Overlapping factor}

The LIMBO process with established process parameters had been repeatedly conducted for 15 times to measure the geometrical value of the LIMBO weld joint and to determine the overlapping factor. The determined mean values to calculate the overlapping factor are the upper side of solidified melt pool diameter with $\mathrm{d}_{\mathrm{t}}=427 \pm 39 \mu \mathrm{m}$ and the weld joint diameter with $\mathrm{d}_{\mathrm{j}}=189 \pm 53 \mu \mathrm{m}$ respectively.

According to this measured value and newly defined overlapping factor in sub-section 3.4, the overlapping factor $\mathrm{O}_{\mathrm{f}}=0 \%$ signifies that the upper side of the solidified melt pool on the interconnector caused by each LIMBO process does not overlap. For $\mathrm{O}_{\mathrm{f}}=25 \%$ the melt pool from the overlap pulsed welding would overlap with this solidified melt pool from the single LIMBO process, however, with the question of whether continuously expanded weld joint is guaranteed through the overlap pulsed welding. Due to the ratio between the upper side of solidified melt pool diameter of interconnector and the weld joint diameter of the single LIMBO process from the previous section, it is expected that the weld joint is continuously expanded from the overlapping factor $\mathrm{O}_{\mathrm{f}}=50 \%$. The simultaneous irradiation of the connection and solidified melt pool is investigated to clarify whether the expansion of weld joint area in case of the overlapping factor $\mathrm{O}_{\mathrm{f}}=75 \%$ is possible or not without destructing the underlying substrate.

\subsection{Pre-heating stage}

The goal of the pre-heating stage during the overlap pulsed welding process is to prepare a lens-like melt at the lower side of the interconnector reproducibly. In order to form a comparable volume of melt during the overlap pulsed welding, same process parameters in despite of the pulse duration are applied for all investigated overlapping factors. Each conducted overlap pulsed welding process is examined with a high-speed camera videography (refer to sub-section 4.2) and the moment of lens-like melt formation $t_{m}$ is shown in figure 9 dependent to overlapping factors. This pre-heating stage is performed without the underlying joining partner (blind welding) and therefore a weld joint between both joining partners is not given for this overlap pulsed welding process.

For all overlapping factors a clear melt formation is observed and the moment of melt formation occurs at least up to four overlap pulsed welding comparably in the reproducible time range. Furthermore, the standard deviation value of the melt occurrence is also comparable for all four overlap pulsed welding despite of third overlap pulsed welding with overlapping factor $\mathrm{O}_{\mathrm{f}}=75 \%$. The overlapping factor $\mathrm{O}_{\mathrm{f}}=50 \%$ shows the most stable result regarding to the moment of melt lens formation despite of a slight decreasing tendency between the second and third overlap pulsed welding process. This slight reduction of the melt formation moment occurs for all investigated overlapping factors and this can be explained with the heat accumulation effect.

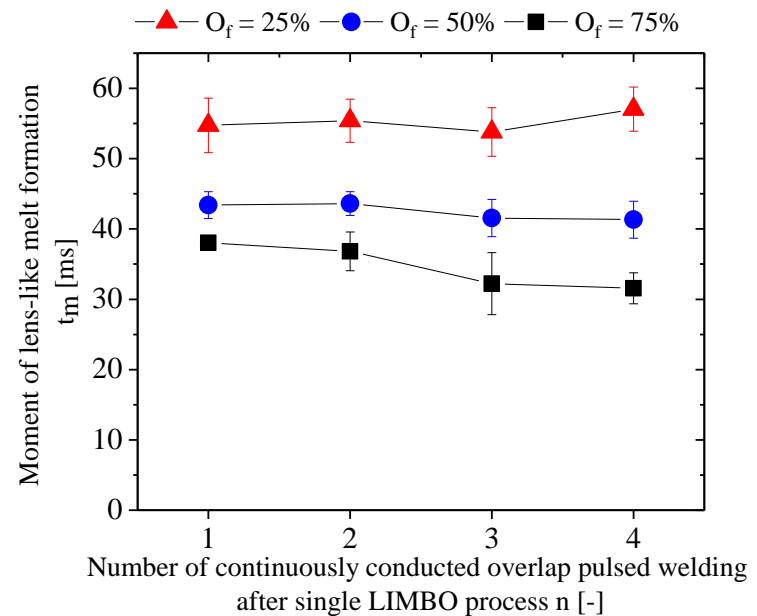

Fig. 9 Moment of lens-like melt formation during the overlap pulsed welding (continuously)

The reduction of the pre-heating stage duration is observed in figure 9 with an increasing overlapping factor. Dependent to the overlapping factor, the irradiated surface area for the following overlap pulsed welding varies between solidified melt pool from the previously welded area and non-melted surface area. According to the overlapping factor equation in sub-section 3.4, the irradiated surface area for the following overlap pulsed welding on the solidified melt pool enhances with the increased overlapping factor. Under an assumption that the geometrical value of the weld after the LIMBO process remains equal for each following overlap pulsed welding, a constant irradiated surface area depending on the overlapping factor can be defined. Taking into account of different absorption rate from solidified melt pool and non-melted surface of interconnector, the total absorption rate in pre-heating stage for overlap pulsed welding enhances with increasing overlapping factor. To investigate this assumption, the top view of blind overlap pulsed welded surface with all overlapping factor is shown in figure 10 .

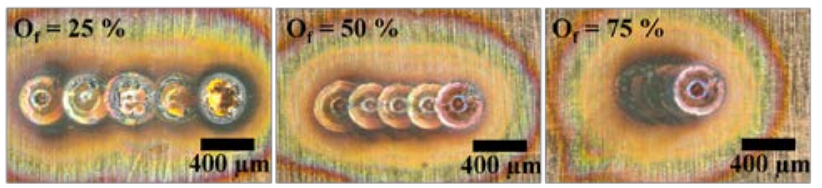

Fig. 10 Top view of blind overlap pulsed welded interconnector

The solidification boarder of melt pool for each single LIMBO process through the overlap pulsed welding process is clearly defined. This clear solidification boarder represents that the solidified melt pool from previous overlap pulsed welding is not remolten completely during the following overlap pulsed welding for all investigated overlapping factors. As for the geometrical value, the solidified melt pool diameter of applied overlap pulsed welding for all overlapping factors are comparably equal. The highest standard deviation value regarding the solidified melt pool diameter is given for the overlapping factor $\mathrm{O}_{\mathrm{f}}=50 \%$ with $\sigma_{\mathrm{t}}=15.1 \mu \mathrm{m}$.

Furthermore, the formation of temper color in an oval form is observed. After the measurement of the temper 
color area, the result shows that the radiation of laser beam during the overlap pulsed welding is applied for investigated overlapping factors within the temper color region. The width of temper color stays relative constant, whereas the length of the temper color increases in the welding direction.

To sum up the pre-heating stage of the overlap pulsed welding process, the irradiated surface area for each overlapping factor can be defined due to the comparable geometrical value of applied overlap pulsed welding process. As for the moment of lens-like melt formation during the overlap pulsed welding, the experiment shows nearly equal results and it divers only in range of the standard deviation.

\subsection{Deflection stage}

After a melt formation at lower side of the upper joining partner, the deflected melt should bridge the gap between both joining partners subsequently during the overlap pulsed welding. This melt deflection should lead to a weld joint area expansion. However, the outgoing position of the melt deflection is for the overlap pulsed welding different to the single LIMBO process, as it is described in previous sub-section 5.1 due to an already existing a weld joint. Despite of this, the deflection stage for the overlap welding process is examined with same process parameters as for the single LIMBO process since the solidified melt pool diameters are comparable (refer to figure 10). The same focal lens adjustment $(\Delta \mathrm{z}=0.5 \mathrm{~mm})$ is used for overlap pulsed welding process, since the modulated laser beam intensity with determined focal lens adjustment value in the deflection stage for the LIMBO process (refer to subsection 4.2) is sufficient to overcome the gap distance $\mathrm{a}=100 \mu \mathrm{m}$.

The duration of the laser beam during the deflection stage is enhanced with a holding time $t_{\mathrm{HT}}$ to investigate the melt deflection behavior over a gap distance $\mathrm{a}=100 \mu \mathrm{m}$. The holding time is defined as an additional laser beam emission in the deflection stage after reaching the adjusted focal lens value $(\Delta \mathrm{z}=0.5 \mathrm{~mm})$. During the laser beam emission in holding time, the intensity of the laser beam stays constant and therefore the temporal modulation of the laser beam is not given anymore. Since the laser beam duration in the deflection stage with the adjustment of the focal lens $\Delta \mathrm{z}=0.5 \mathrm{~mm}$ is $\mathrm{t}=5 \mathrm{~ms}$ (refer to sub-section 3.2 ), the holding time for the deflection stage starts therefore after the duration of the deflection stage ( $t=5 \mathrm{~ms}$ ).

The moment of maximum melt deflection in deflection stage dependent to the overlapping factor with an additional holding time is shown in figure 11. Only one following overlap pulsed welding after the LIMBO process is applied for this experiment.

The result for the overlapping factor $\mathrm{O}_{\mathrm{f}}=25 \%$ shows a comparable melt deflection behavior to the LIMBO process respectively. This result can be interpreted that the overlap pulsed welding with the overlapping factor $\mathrm{O}_{\mathrm{f}}=25 \%$ is not influenced by the previously conducted LIMBO process. Regarding the deflection stage, the interaction between each conducted weld joint is nearly given and this outcome indicates that the overlapping factor $\mathrm{O}_{\mathrm{f}}=25 \%$ would not lead to a continuously expanded weld joint area after the overlap pulsed welding process. On the other hand, the overlapping factors from $\mathrm{O}_{\mathrm{f}}=50 \%$ require an additional laser beam emission in order to deflect the melt over a defined gap distance and this is apparently affected by already existing weld joint. It is observed that the duration of deflection stage enhances with higher overlapping factor This results shows that the sufficient melt deflection of the overlap pulsed welding is possible with enhancement of laser beam intensity and energy by extending the deflection stage with a certain holding time $t_{\mathrm{HT}}$.

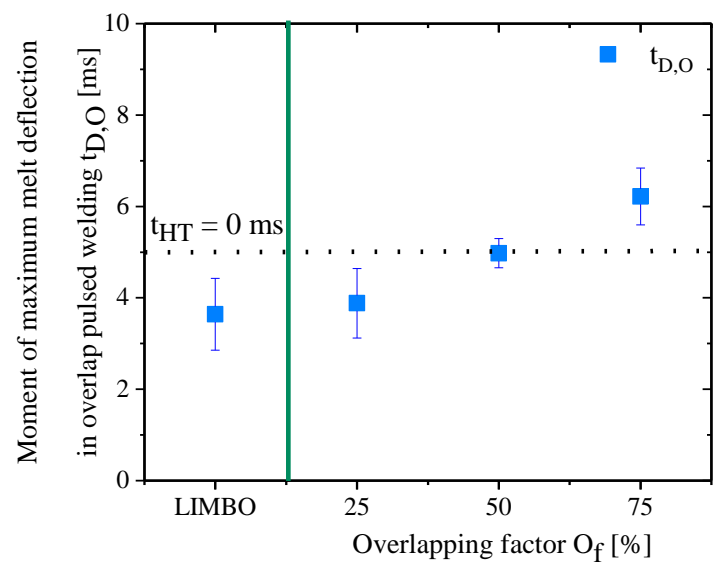

Fig. 11 Moment of maximum melt deflection in overlap pulsed welding

The increasement of overlapping factor leads to higher demands on laser beam intensity and energy to deflect the melt over a gap distance $\mathrm{a}=100 \mu \mathrm{m}$ during the overlap pulsed welding. The possible explanation is the change of the heat conduction condition due to the connection between both joining partners. With increasing overlapping factor, the laser beam is irradiated progressively near and even partly above the previously applied weld joint which favors the heat flow condition into the underlying substrate. Due to the heat loss, the duration of deflection stage should be extended with the holding time to supply sufficient energy input.

\subsection{Overlap pulsed welding with the underlying join- ing partner}

The weld joint expansion conducted with the LIMBO process should not exceed the destruction threshold for the substrate under the thin copper layer while the weld joint is expanded. In order to minimize the heat accumulation effect on each conducted overlap pulsed welding process, a cooling time $t_{c}=1.5 \mathrm{~s}$ between each overlap pulsed welding is implemented. This cooling time is implemented to compromise the additional laser beam energy input through the holding time. Further investigation on the heat accumulation effect is not performed.

The shadow projection image sequence of the deflection and the joining stage for one following overlap pulsed welding with the overlapping factor $\mathrm{O}_{\mathrm{f}}=50 \%$ is shown in figure 12 and the description of the shadow projection image is equal to figure 6 . 


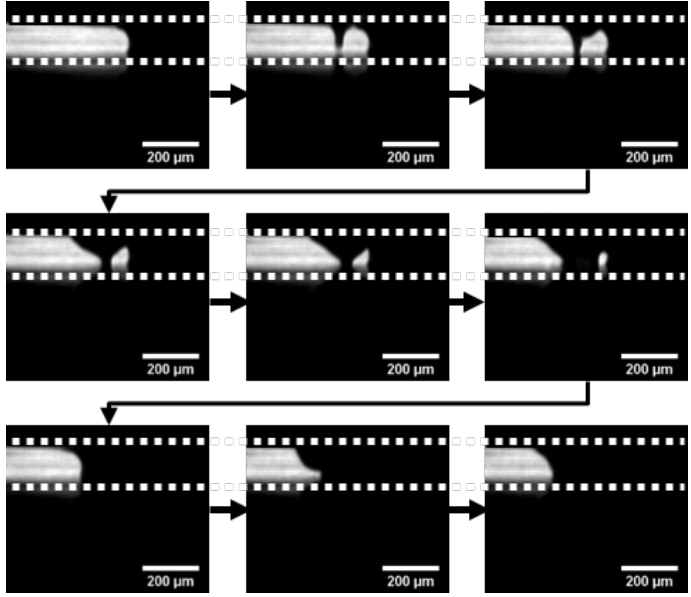

Fig. 12 Shadow projection image sequence of the overlap pulsed welding process with $\mathrm{O}_{\mathrm{f}}=50 \%$ (time flow in arrow direction)

The melt is deflected outside of the weld joint between both joining partners and connects on the surface of the copper layer. After bridging the gap with the deflected melt due to the increasement of the intensity and the further energy input of the laser beam during the holding time, the volume of the deflected melt increases over the time. With increasing deflected melt volume and the melt dynamic, the gap between the already performed weld joint and the deflected melt volume closes. This procedure may leads to a continuously expanded weld joint.

With given experimental settings and determined process parameters, the overlap pulsed welding with the overlapping factor $\mathrm{O}_{\mathrm{f}}=50 \%$ is conducted and the result is shown in figure 13 below. In middle of the expanded weld joint area, there is a possible void between the first and second overlap pulsed welding. To determine whether this void is a gap or a pore, the expanded weld joint area should be tested on the density measurement. However, this test is not further investigated within this paper.

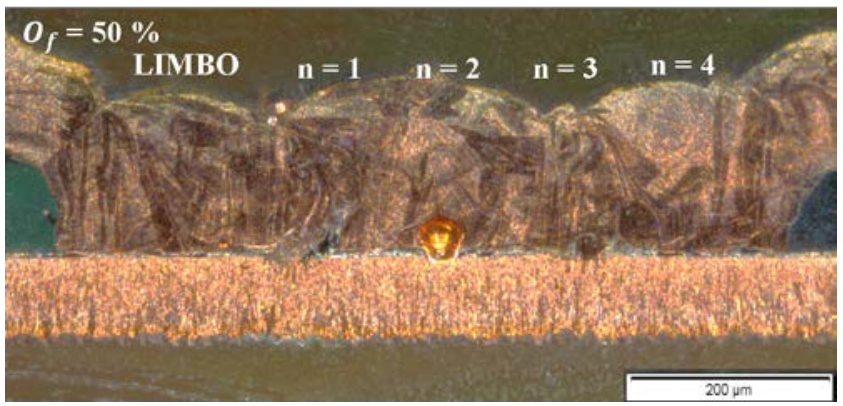

Fig. 13 Expanded weld joint area with overlap pulsed welding process

A successful expansion of the LIMBO process is proceeded with non-damaged on the thermally sensitive substrate and expanded weld joint The length of this expanded weld joint is $l=1.02 \mathrm{~mm}$ and for each overlap pulsed welding the area has increased in $\Delta \mathrm{d}=205 \pm 23 \mu \mathrm{m}$ steps.

\section{Conclusion}

The expansion of weld joint with the LIMBO process is applied between a copper interconnector with thickness $\mathrm{h}=200 \mu \mathrm{m}$ and copper layer with thickness $\mathrm{h}=100 \mu \mathrm{m}$ without destructing the thermally sensitive substrate underneath. In this paper, the temporal modulation of laser beam spot size is applied which is used to deflect the melt pool to the lower joining partner. Furthermore, the reproducibility of the LIMBO process is investigated for the pre-heating and deflection stages separately. As for the result of reproducibility, the LIMBO process is expanded with a prepulsing stage to pre-oxidize the upper joining partner. The pre-pulse stage is conducted with the same laser beam source which is also used for the following LIMBO process. The melt dynamic in deflection stage is examined with a high speed camera shadow videography. The adjustment range for the focal lens is set to $\Delta \mathrm{z}=0.5 \mathrm{~mm}$ to deflect the melt pool over the gap $\mathrm{a}=100 \mu \mathrm{m}$ and to minimize the residence time of melt on the surface of thin copper layer.

Based on the reproducible outgoing position for the LIMBO process, the overlapping factor is determined with solidified melt pool on the interconnector with the diameter $\mathrm{d}_{\mathrm{t}}=427 \pm 39 \mu \mathrm{m}$. The overlap pulsed welding of the LIMBO process is characterized dependent to overlapping factor and number of applied overlap pulsed welding process in order to achieve continuously expanded weld joint without damaging the underlying substrate. The duration of pre-heating stage reduces with increasing overlapping factor and this duration is independent to the number of conducted at least up to four following overlap pulsed welding. The duration of deflection stage increases with increasing overlapping factor due to the change of the heat conduction condition caused by the existence of previously applied weld joint between both joining partners.

The overlapping factor $\mathrm{O}_{\mathrm{f}}=50 \%$ and determined process parameters with a cool time $t_{c}=1.5 \mathrm{~s}$ lead to continuously expanded weld joint over a $l=1 \mathrm{~mm}$ with laser-gap welding method.

\section{Outlook}

The next step for this study is to investigate the heat accumulation effect dependent to the overlapping factor, so the necessary cooling time between each pulse can be determined. For this purpose, a heat flow simulation during the overlap pulsed welding is required. Throughout the simulation of the overlap pulsed welding, the penetration depth of the deflected melt into the underlying copper layer should be also investigated. This simulation result could be used to conclude the lowest thickness limit of the underlying copper layer for the LIMBO process applications.

Also, the void in middle of the expanded the weld joint area of the LIMBO process should also be evaluated. The aim of the overlap pulsed welding process is to achieve a continuously expanded weld joint area and this also should lead to hermitically dense weld joint. The hermitically dense weld joint will allow a homogenous current flow into the underlying copper layer and lower the electrical resistance.

As for the further step, the formula to calculate the overlapping factor should be extended with the temporal terms. To extend this formula, the correlation and influence of following terms should be further investigated:

- determination of the cooling time $t_{c}$ between each overlap pulsed welding, 
- accurate steering and control of the focal lens in deflection stage,

- influence of the number of conducted overlap pulsed welding with $\mathrm{n}>4$,

- unification of the divided stages of the LIMBO process.

The open investigation points for this paper is evaluation of the mechanical and electrical properties of the expanded weld joint diameter.

\section{Acknowledgment}

This work was supported by the Federal Ministry for Economic Affairs and Energy in the frame of the FlexJoin project under the contract number 01MX15010B.

\section{References}

[1] T. Gottwald and C. Rößle: The PCB Magazine, 5, (2015) p.52.

[2] T.K. Hooghan, S. Nakahara, K. Hooghan, R. W. Privette, M.A. Bachman, R.S. Moyer: Thin Solid Films, 437, (2003) 235.

[3] Z.W. Zhong: Microelectron. Reliab. 51 (2011) 4.

[4] K.S. Siow: J. of Electron. Mater., 43 (2014) 947.

[5] S. W. Britten: Laser Tech. J, 2, (2016) 53.

[6] D. K. Dijken, W. Hoving, J. Th. M. De Hosseon: J. Laser Appl., 15, (2003) 11.

[7] S. Britten, S. Wein, A. Olowinsky, A. Gillner: PCIM Europe, Nuremberg, (2015) p.1321.

[8] M.J. Torkamany, M.J. Hamedi, F. Malek, J. Sabbaghzadeh: J. Phys. D: Appl. Phys., 39, (2006) 4563.

[9] S. W. Britten: "Bauteilschonende Verbindungstechnik auf Metallisierungen durch moduliertes Laserstrahlschweißen” ed. by Apprimus Verlag (Publisher, Aachen, 2017) p.101.

[10] A. Blom, P. Dunias, P. van Engen, W. Hoving, J. Kramer: Proc. Vol. 4977 on High-Power Lasers and Applications, San Jose, (2003) p.493.

(Received: June 24, 2018, Accepted: September 12, 2018) 